\title{
Mise En Évidence De Nouvelles Structures Géologiques Dans La Région De Brobo (Centre De La Côte d'Ivoire). Aide À La Compréhension De La Tectonique Du Paléoprotérozoïque Du Craton Ouest Africain
}

\author{
Daï Bi Seydou Mathurin, \\ Ouattara Gbele,
} Koffi Gnammytchet Barthélémy, Laboratoire des Géosciences, Cadre de vie, Environnement et Sciences Géographique, UMRI 68 - Ecole Doctorale Polytechnique, Institut National

Polytechnique Félix Houphouët-Boigny, Yamoussoukro, Côte d'Ivoire

\section{Gnanzou Allou, Coulibaly Inza,}

Laboratoire de Géologie du Socle et Métallogénie, UFR STRM,

Université Félix Houphouët-Boigny, Côte d'Ivoire

Doi: 10.19044/esj.2018.v14n18p305 URL:http://dx.doi.org/10.19044/esj.2018.v14n18p305

Abstract

The lithological and structural observations of the region of Brobo (Central Côte d'Ivoire) indicate a succession of metasedimentary rocks (micaschists with cordierite, silstones, graphitic sediments, sandstones with amphibole-garnet, etc.) intermixed with volcanic rocks (rhyolite, dacite, andesite, basalt and the volcanoclastics). The whole is intruded by granites with one or two micas, sometimes porphyries, granodiorites, gabbros, and granite gneisses. Interpretations of Landsat $\mathrm{ETM}^{+}$, RadarSat-1 and SRTM remote sensing imageries, as well as field data, revealed several lineament directions which, after field control, correspond to major faults and shear zones. These large structures show the N-S, NE-SW, NNE-SSW, E-W, NWSE, and NNW-SSE orientations. The field data also made it possible to describe several structures and to propose a preliminary geodynamic model for the setting and structuring of the formations of this region. This model suggests that the geodynamic took place in three stages: distension with a deformation of basement formations generating a gneissocity (D1), as well as deposits of sediments in the basins; followed by a NW-SE to E-W convergence generating a cleavage in the volcanogenic series (D2). This phase of deformation continues while creating, locally, a strain slip cleavage or a 
transposed schistosity. The third cleavage affects the volcanogenic series (fractures cleavages, D3) and ends in large corridors of ductile shear zones and associated faults.

Keywords: Lithostructural mapping, Remote Sensing Imagery, Birimian, Brobo, Central Côte d'Ivoire

\section{Résumé}

Les observations lithologiques et structurales de la région de Brobo (Centre de la Côte d'Ivoire) indiquent une succession de roches métasédimentaires (micaschistes à cordiérite, pélites, sédiments-graphiteux, grès à amphibole-grenat,...) intercalées par un ensemble de roches volcaniques (rhyolite, dacite, andésite, basalte et les volcanoclastiques). L'ensemble est intrudé par des granites à un ou deux mica(s) parfois porphyroïdes, de granodiorites, de gabbros et de gneiss granitiques. Les interprétations des images satellitales Landsat ETM+ ${ }^{+}$, RadarSat-1 et SRTM, ainsi que les données de terrain, ont permis de relever plusieurs directions de linéaments qui, après contrôle sur le terrain, correspondent à des zones de fractures et failles majeures. Ces grandes structures montrent les orientations N-S, NE-SO, NNE-SSO, E-O, NO-SE et NNO-SSE.Les données de terrain ont également permis de décrire plusieurs structures et de proposer un modèle géodynamique préliminaire pour la mise en place et la structuration des formations de cette région. Ce modèle suggère que la structuration s'est déroulée en trois étapes : distension avec une déformation du socle générant une foliation (D1), ainsi que des dépôts de sédiments dans les bassins ; suivie d'une convergence NO-SE à E-O engendrant une schistosité de flux dans la série volcano-sédimentaire (D2). Cette phase de déformation se poursuit en créant, localement, une schistosité de crénulation. Une troisième schistosité affecte la série volcano-sédimentaire (schistosité de fractures, D3) et se terminant par de grands couloirs de décrochements ductiles et failles associées.

Mots-clés: Cartographie Lithostructurale, Imagerie Satellitale, Birimien, Brobo, Centre Côte d'Ivoire

\section{Introduction}

Les roches vertes birimiennes d'Afrique de l'Ouest sont constituées de ceintures de roches métavolcaniques et métasédimentaires. Ces formations sont intrudées par des plutons de granites calco-alcalins et alcalins. Il est également à noter que les formations paléoprotérozoïques (birimiennes) de l'Afrique de l'Ouest sont bien connues pour accueillir de nombreuses minéralisations aurifères économiques (Milési et al., 1989, 1992 ; Kusnir, 
1999 ; BRGM, 2005 ; Assié, 2008 ; Béziat et al., 2008 ; Billa \& Bonnemaison, 2009 ; Dabo, 2011 ; Houssou, 2013 ; Gnanzou, 2014 ; Ouattara, 2015 ; Ouattara et al., 2016 ; Houssou et al., 2017 ; Traoré, 2017).

La région de Brobo (Centre de la Côte d'Ivoire) fait partie du sillon d'Oumé-Toumodi-Fetêkro (Figure 1), qui renferme dans sa partie sud les mines de Bonikro, Hiré (exploitées par la Société Newcrest Mining), ainsi que le gite aurifère de Dougbafla et la mine d'Agbahou (exploitée par la Société Endeavour CI). Cependant, les parties nord et centrale du sillon restent encore peu étudiées.

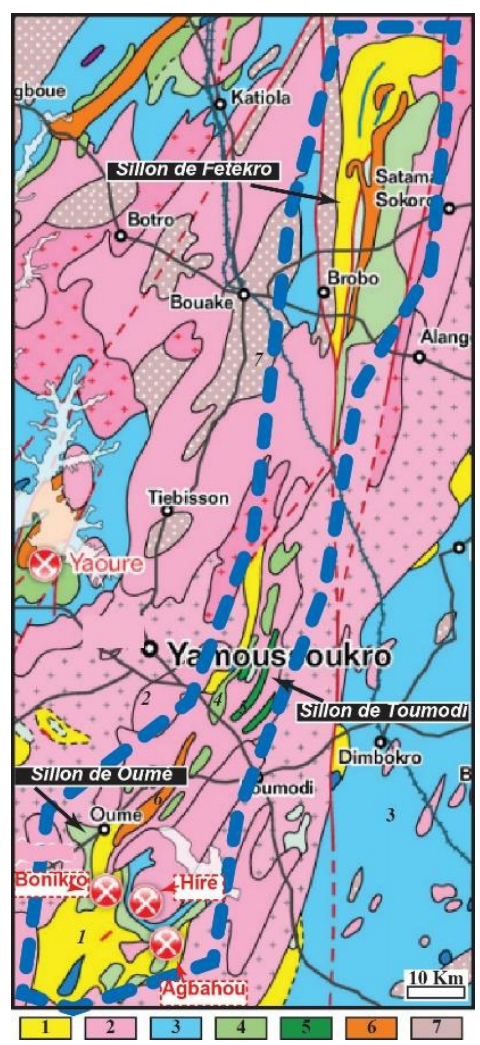

Figure 1. Carte géologique du sillon de Oumé-Toumodi-Fetêkro (modifié selon Sems Exploration, 2017). 1= roches volcaniques felsiques et dykes ; 2= granitö̈des indifférenciés ; $3=$ roches vocaniques indifférenciées et volcano-sédiments ; $4=$ roches basiques $; 5=$ rhyodacites et rhyolites $; 6=$ formations fluviodeltaïques de type Tarkwaien ; $7=$ leucogranites.

Les travaux antérieurs, portant sur la lithologie et les structures géologiques, ont concerné la partie Nord de la zone d'étude (Lemoine et al., 1985 ; Lemoine, 1988 ; Adou et al., 1990 ; Leake, 1992 ; Yao, 1998 ; Adou, 2000 ; Gnanzou, 2014 ; Gnanzou et al., 2015).

Au plan lithologique, à partir de la carte géologique existante (Yao, 1988), plusieurs formations géologiques ont été cartographiées (Figure 2) : 
granitoïdes variés (granites à deux micas, granites à biotite, granodiorites, gabbros, diorites, gneiss), métavolcanites (surtout des pyroclastites acides ou basiques) et des métasédiments (micaschistes, schistes, grauwackes, quartzites).

$\mathrm{Au}$ plan structural, quelques structures régionales ont été cartographiées: faille subméridienne de N'Zi-Brobo, failles NE-SO de Dimbokro, NO-SE et E-O. Notre travaille permettra de préciser le tracé de ces structures et cartographier de nouvelles.

Notre travail vise donc à apporter de nouvelles données lithologiques, mais surtout structurales dans la région de Brobo ; cela permettra de mieux comprendre la structuration pour une meilleure approche de l'évolution tectonique de cette zone du craton ouest-africain.

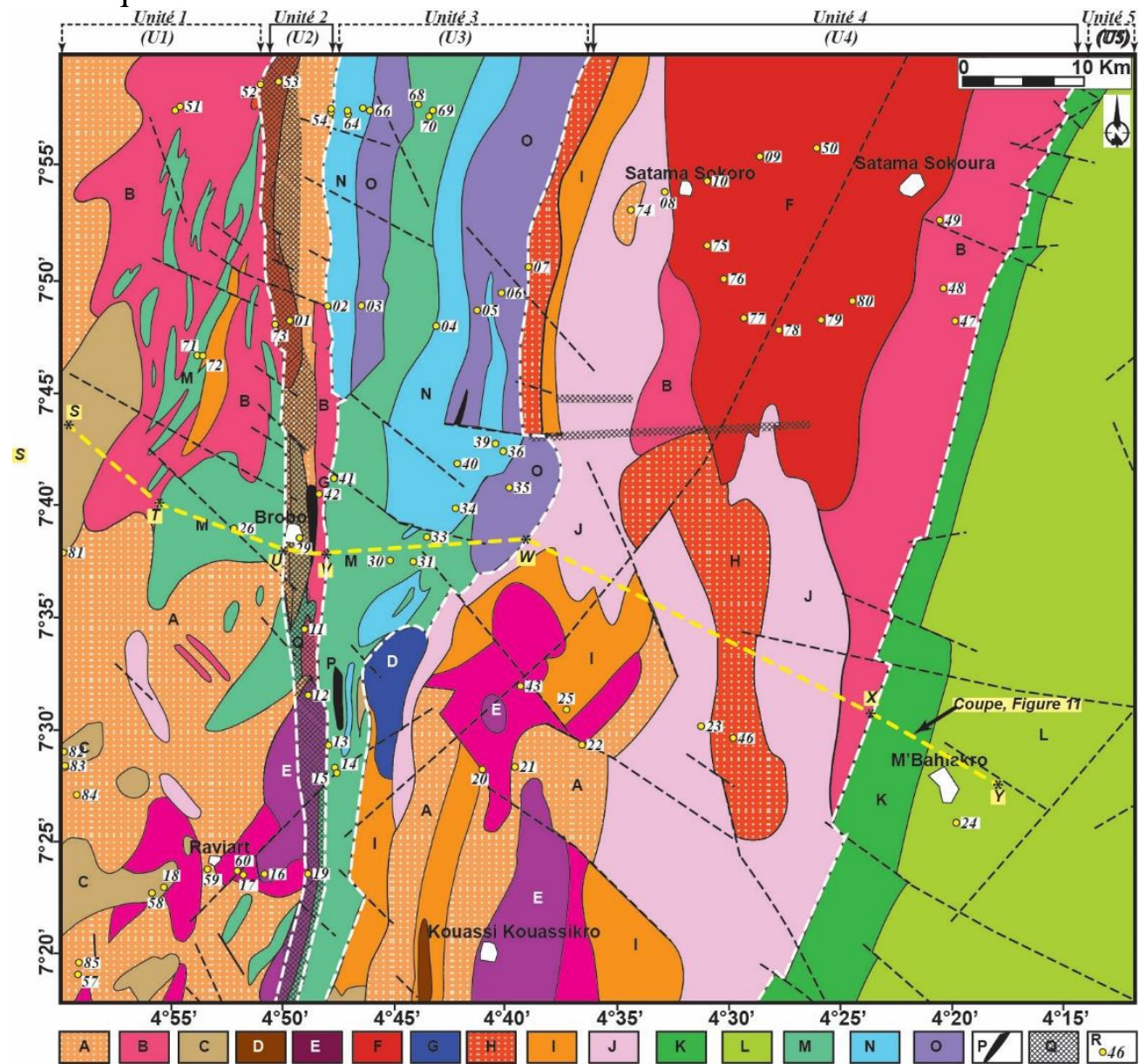

Figure 2. Extrait de la carte géologique de la feuille de M’Bahiakro (Yao, 1988), modifiée. $\mathrm{A}=$ granite hétérogène $; \mathrm{B}=$ granodiorite orientée à biotite ou biotite-amphibole $; \mathrm{C}=$ granite à biotite-muscovite; $\mathrm{D}=$ granodiorite à biotite-amphibole ; $\mathrm{E}=$ granite leucocrate à biotite ; $\mathrm{F}=$ granodiorite porphyroïde à biotite-amphibole $; \mathrm{G}=$ granodiorite porphyroïde orienté à

biotite-amphibole $; \mathrm{H}=$ gneiss migmatitique $; \mathrm{I}=$ gneiss migmatitique $; \mathrm{J}=$ migmatite rubanée ; Série de la moyenne Comoé : $\mathrm{K}=$ schiste noir, grauwacke, pélite gréseuse ; $\mathrm{L}=$ 
grès, grès schisteux, schiste ; $\mathrm{M}=$ micaschiste ; $\mathrm{N}=$ lave acide, pyroclastite, tuf acide , métagrauwacke ; $\mathrm{O}=$ pyroclastite, brêche, tuf basique ; $\mathrm{P}=$ quartzite $; \mathrm{Q}=$ mylonite $; \mathrm{R}=$ échantillon. Provinces lithologiques (U1, U2, U3, U4 et U5). De S à Y : coupe interprétative de la Figure 11.

\section{Matériel et Méthodes}

\section{Matériel}

Ce travail utilise l'imagerie Landsat $7 \mathrm{ETM}^{+}$(Figure 3), l'imagerie RadarSat-1 (Figure 4) et l'imagerie SRTM (Figure 5) pour la cartographie lithologique et structurale de la région de Brobo. Leurs interprétations permettront la mise à jour partielle de la carte géologique de la feuille du degré carré de M'Bahiakro. Les images utilisées ont les caractéristiques suivantes (Tableau 1) :

Tableau 1. Caractéristiques des images utilisées.

\begin{tabular}{|c|}
\hline Images Landsat ETM ${ }^{+}$orthorectifiées Geo-Tiff \\
\hline Date d'acquisition : 07 Février 2011 \\
\hline Path/Row : 196/055 \\
\hline Nombre de canaux (bandes) : 9 bandes (B1, B2, B3, B4,B5, B6.1, B6.2, B7 et B8) \\
\hline Résolution : 30m pour B1, B2, B3, B4, B5 et B7 \\
\hline $57 \mathrm{~m}$ pour $\mathrm{B} 6.1$ et $\mathrm{B} 6.2$ \\
\hline $14,25 \mathrm{~m}$ pour $\mathrm{B} 8$ \\
\hline Système de Projection : UTM, Zone $30 \mathrm{~N}$ \\
\hline Datum : WGS-84 \\
\hline $\begin{array}{ll}\text { B1: bleu; B2: vert; B3: rouge, B4: proche infrarouge; B5: } & \begin{array}{l}\text { Caractéristiques spectrales : } \\
\text { infrarouge moyen; B6: infrarouge thermique; B7: } \\
\text { infrarouge lointain; B8: panchromatique }\end{array}\end{array}$ \\
\hline Image RadarSat 1 \\
\hline Antenne active : Bande $\mathrm{C}$ \\
\hline Fréquence centrale : $15,3 \mathrm{GHz} 96 / 055$ \\
\hline Largeur de bande: $30 \mathrm{MHz}$ \\
\hline Polarisation : $\mathrm{HH}$ \\
\hline $\begin{array}{l}\text { ScanSAR étroit (Largeur de } \\
\text { fauchée nominale }(\mathrm{km}):\end{array}$ \\
\hline Résolution nominale $50 \mathrm{~m}$ \\
\hline Système de Projection : UTM, Zone 30N \\
\hline Datum : WGS-84 \\
\hline Images SRTM (Shuttle Radar Topography Mission) \\
\hline Bandes du capteur SRTM : Bande C et bande X \\
\hline Résolution : $90 \mathrm{~m}$ \\
\hline Couverture : $60^{\circ}$ nord et $56^{\circ}$ sud \\
\hline Système de Projection : UTM, Zone $30 \mathrm{~N}$ \\
\hline Datum : WGS-84 \\
\hline
\end{tabular}




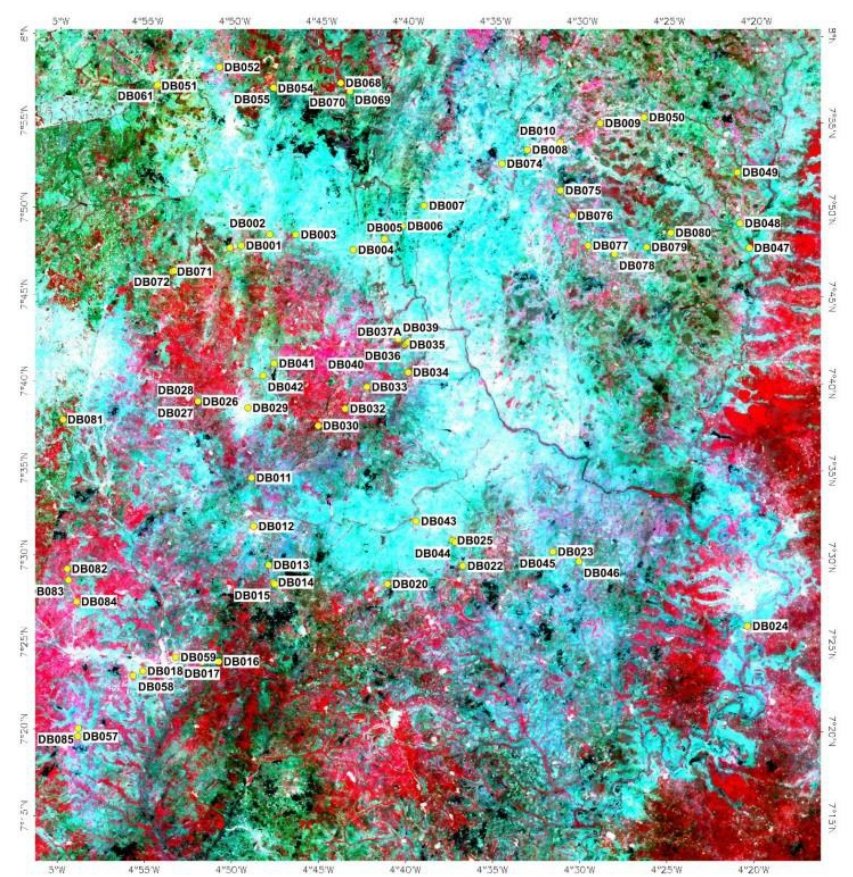

Figure 3. Image Landsat $7 \mathrm{ETM}^{+}$, en composition colorée 432 et affleurements.

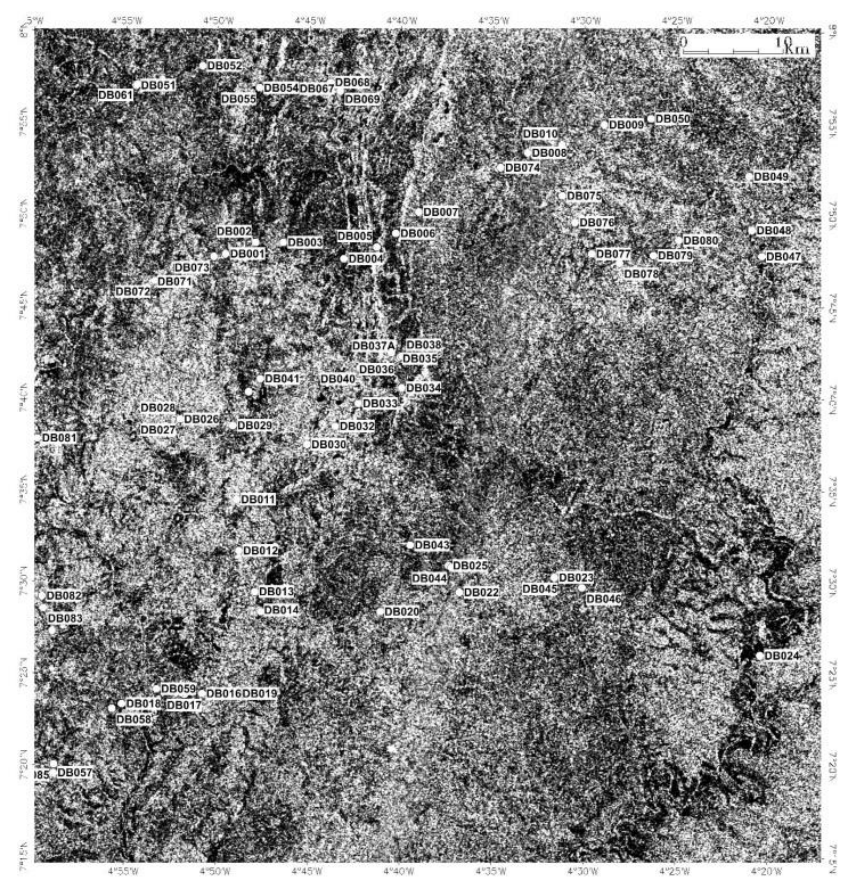

Figure 4. Image RadarSat-1 de la région de Brobo et affleurements. 


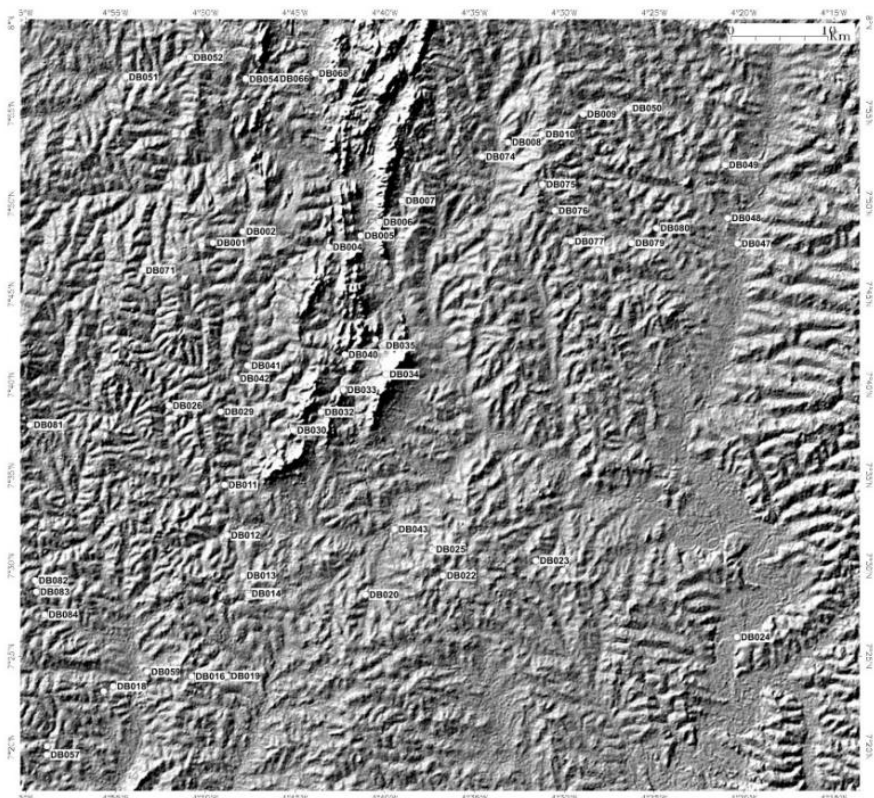

Figure 5. Image SRTM rehaussée Brobo et affleurements.

\section{Méthodes}

Les images Landsat $\mathrm{ETM}^{+}$ayant été préalablement géoréférencées et orthorectifiées, nous avons procédé à des traitements aboutissant à des images en composition colorées. La combinaison des canaux 4, 3, 2 permet d'avoir une image plus lisible et plus contrastée. Les images RadarSat-1 et SRTM ont subi différentes méthodes d'amélioration des contrastes et de rehaussement.

Les interprétations analogiques ont consisté en l'extraction des linéaments, en la détermination des ensembles géologiques (en tenant compte des teintes et des textures sur l'image) et en l'extraction du réseau hydrographique sur support papier. Pour l'analyse numérique, nous avons procédé au rehaussement des structures linéaires en utilisant des filtres directionnels (Himyari et al., 2002; Jourda et al., 2006; Ta et al., 2008; Djemai et al., 2009; Guergou \& Amri, 2009; Kouamé et al., 2009). Les matrices 7x7 de ces filtres sont indiquées dans le Tableau 2. Les validations de ces cartes préliminaires ont ensuite été effectuées sur le terrain. La carte définitive obtenue a tenu compte des cartes existantes et des objets géologiques (lithologie et structures observées sur le terrain). Le cheminement de notre démarche est présenté dans la Figure 6. 
Tableau 2. Matrices de quelques filtres directionnels utilisés.

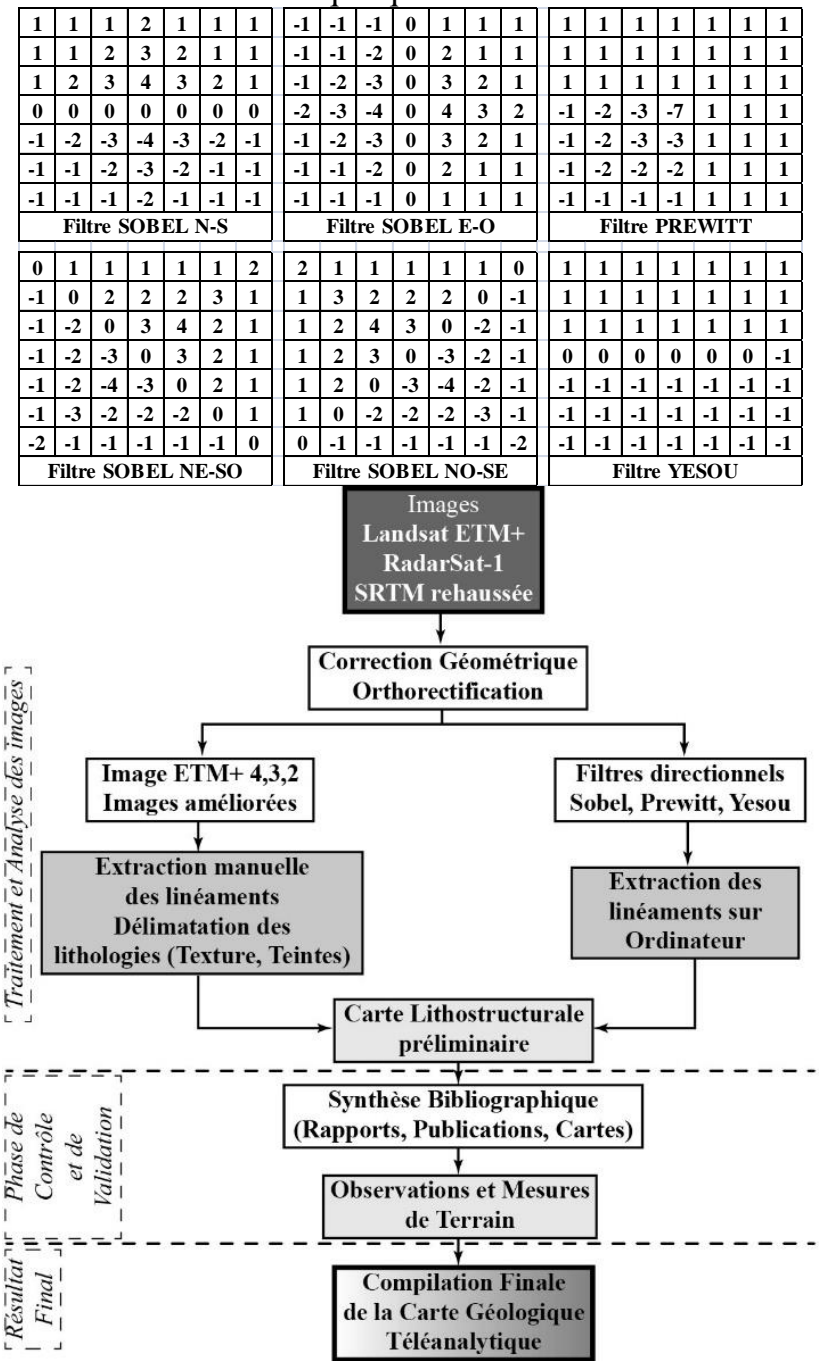

Figure 6. Cheminement des traitements et analyses à partir des images satellitales.

L'interprétation de chaque image et leurs combinaisons ont permis d'avoir une carte de synthèse finale (Figure 7). 


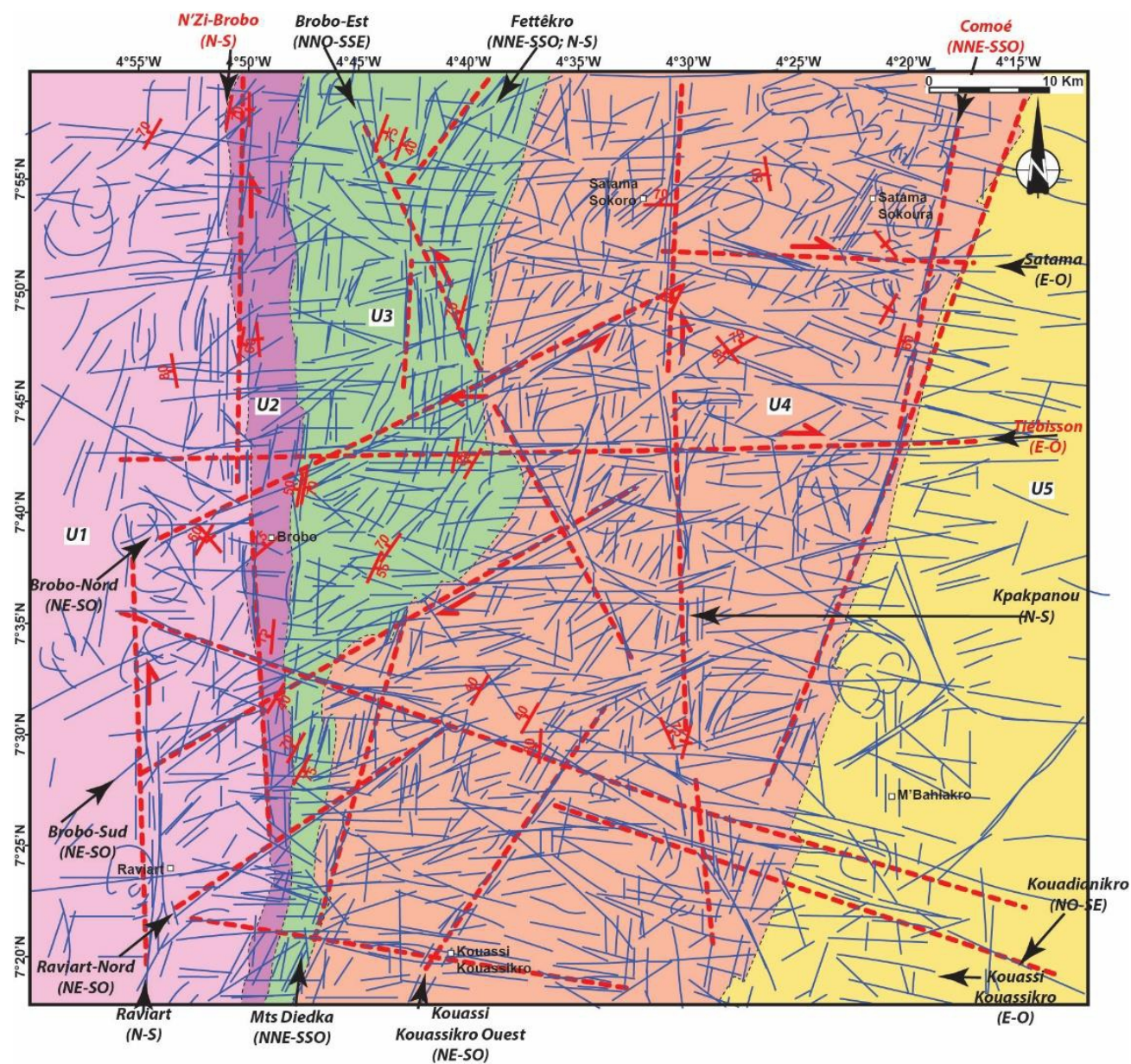

Figure 7. Carte lithostructurale téléanalytique finale. Les noms en rouge indiquent les failles connues sur les cartes antérieures. Provinces lithologiques (U1, U2, U3, U4 et U5).

\section{Résultats}

\section{Cartographie lithologique}

$\mathrm{Au}$ plan lithologique, notre travail permet d'avoir une nouvelle approche lithologique (en tenant compte de la nature et des structures qui affectent ces formations), cela à partir de la carte géologique de Yao (1988). La région de Brobo comporte plusieurs unités lithologiques :

L'Unité 1 (U1): située à l'Ouest; elle est caractérisée par des structures circulaires à subcirculaires. Les formations sont granitoïdiques, avec des lambeaux de métasédiments. Les linéaments sont de faibles longueurs.

L'Unité 2 (U2) : constitue les formations mylonitiques de la faille de Brobo-N'Zi. (Figure 8 G, H). Les linéaments sont fins et orientés N-S. La zone de cisaillement (shear zone) affecte autant les formations de l'Unité 1, que les formations méta-volcanosédimentaires de l'Unité 3. 
L'Unité 3 (U3) : située dans la partie centrale, elle constitue les formations du sillon de Fettêkro. Il s'agit de métasédiments (micaschistes, schistes, quartzites,...), de métavolcanites et de méta-volcanosédiments (basaltes, pyroclastites acides ou basiques), (Figure 8 A, B, C). L'ensemble est par endroits intrudé de gabbros et diorites peu ou pas déformés. Les linéaments sont généralement N-S à NNE-SSO, parfois courbes, avec quelques structures circulaires.

L'Unité 4 (U4) : représentée par l'ensemble granitique depuis Satama (au Nord) jusqu'à Kouassi Kouassikro (au sud). On y rencontre des granites à un ou deux mica(s) parfois porphyroïdes, de granodiorites, de gabbros et de gneiss (Figure 8 D, E, F). Les linéaments sont de diverses directions, avec beaucoup de structures circulaires.

L'Unité 5 (U5): constitue le bassin sédimentaire à l'Est de M'Bahiakro. Ces formations constituent les métasédiments de la partie Ouest du bassin de la moyenne Comoé (Thiéblemont et al., 1987 ; Vidal \& Alric, 1987 ; Vidal et al., 2009 ; Mortimer, 2016). Celle-ci est marquée par très peu de linéaments, qui sont généralement E-O. La zone de contact entre les unités U4 et U5 est marquée par la faille de la Comoé ou Dimbokro de direction NESO (Bessoles, 1977 ; Koita et al., 2010). 

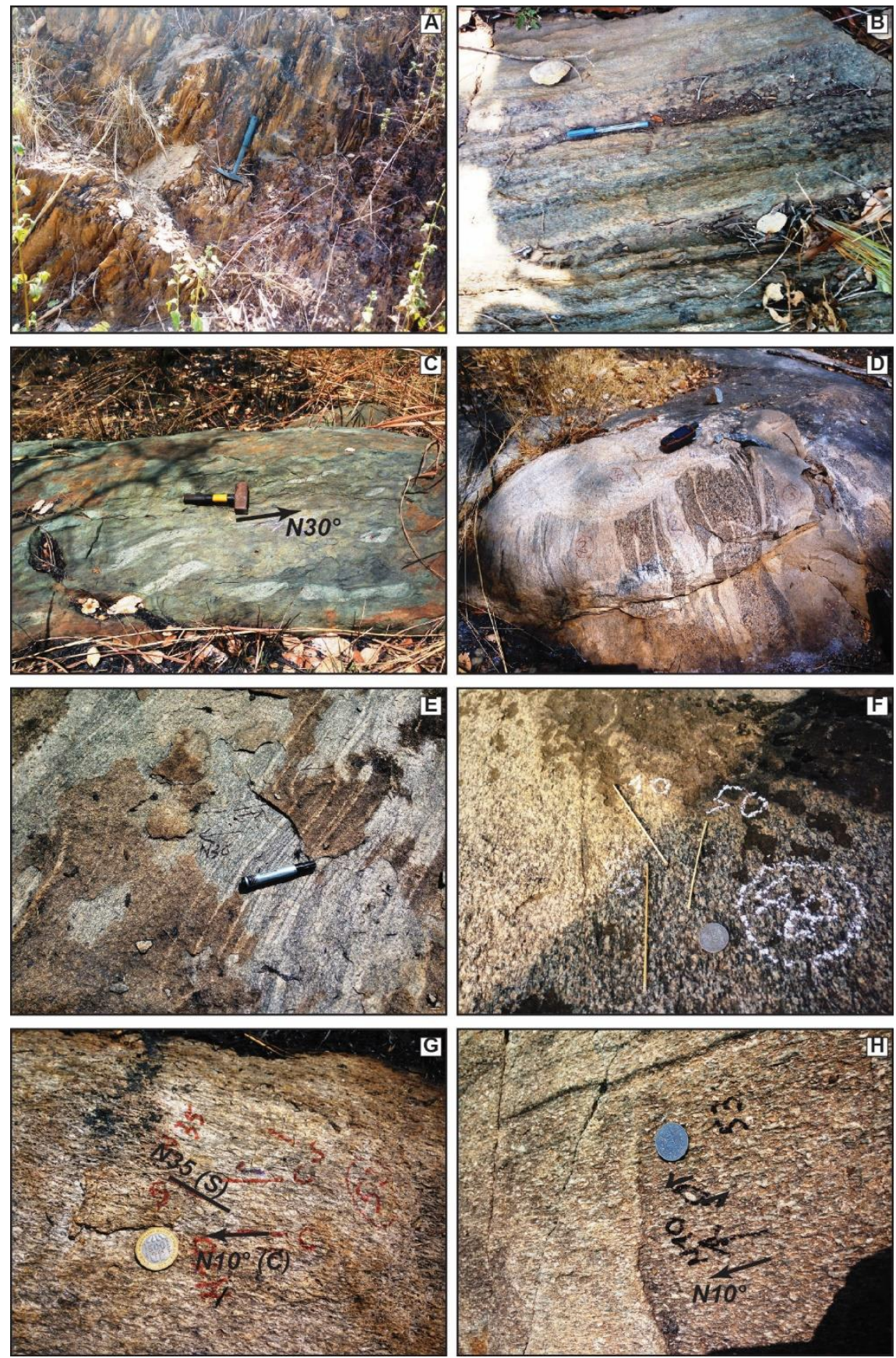

Figure 8. Quelques images d'affleurements. A : Schistes graphiteux ; B : Micaschistes à staurotide ; $\mathrm{C}$ : Métabrèches basiques ; D : Métagranite avec intrusion de leucogranite; $\mathrm{E}$ :

Gneiss granitique à biotite ; F : Granite porphyroïde ; G et $\mathrm{H}:$ Granite mylonitique de Brobo. 


\section{Cartographie structurale}

Les données structurales sont issues de l'interprétation des images satellitales et des données recueillies sur le terrain. Nous avons plusieurs directions de fractures et de failles. Il s'agit des directions : N-S à NNE-SSO; $\mathrm{N} 90^{\circ}$ à $\mathrm{N} 100^{\circ}$; NO-SE à NNO-SSE. Le tracé des failles déjà connues dans la littérature (Lemoine, 1988 ; Leake, 1992 ; Ouattara et al., 2012 ; Gnanzou, 2014 ; Gnanzou et al., 2015) est précisé : failles de Brobo-N'Zi de direction $\mathrm{N}-\mathrm{S}$, de la Comoé ou Dimbokro de direction NNE-SSO et de Tiebisson de direction E-O.

De nouvelles failles sont cartographiées (Figure 7): 1) failles de Brobo-Nord, Kouassi Kouassikro-Ouest, Brobo-Sud, Raviart-Nord de directions NE-SO ; 2) la faille de Fettêkro de direction NNE-SSO montrant dans sa partie sud des flexions, probablement liées aux jeux des failles E-O tardives (failles de Satama, Kouassi Kouassikro) ; 3) faille de Brobo-Est et de Kouadianikro de directions NNO-SSE à NO-SE ; 4) une série de linéaments discontinus de direction $\mathrm{N}-\mathrm{S}$ (faille de Raviart, Kpakpanou; cette dernière correspond à la continuité de l'accident de Katidougou décrit plus au nord par Leake (1992), Gnanzou (2014). Ces dernières sont parallèles à la shear zone de N'Zi-Brobo.

Sur le terrain, différentes structures ont été observées en fonction de la nature de la roche. Les roches métasédimentaires semblent être les plus déformées (Figure 9). En effet, en plus de la schistosité de flux $\left(\mathrm{N} 10^{\circ}\right.$ à $\left.\mathrm{N} 30^{\circ}\right)$ qui est surimposée à la stratification ( $\mathrm{S} 0)$, on note, par endroits, une schistosité de crénulation $\left(\mathrm{N}^{\circ} 0^{\circ}\right.$ à $\left.\mathrm{N} 70^{\circ}\right)$. Une troisième schistosité peut affecter ces roches. Il s'agit d'une schistosité de fractures très régulière $\left(\mathrm{N} 120^{\circ}\right.$ à $\left.\mathrm{N} 130^{\circ}\right)$. L'ensemble de ces structures est recoupé par des fractures et failles dextres ou senestres. Dans les roches granitiques, on note sur certains affleurements une foliation NE-SO. Un rubanement peut apparaître lorsque la déformation est faible. La foliation est parfois plissée mais n'engendrant pas d'autres structures. Les grands couloirs de cisaillement concernent la faille de N'ZiBrobo où les plans $\mathrm{C}\left(\mathrm{N} 160^{\circ}\right)$ et $\mathrm{S}\left(\mathrm{N} 10^{\circ}\right)$ sont bien exprimés avec des plans $\mathrm{C}^{\prime}\left(\mathrm{N} 30^{\circ}\right)$ associés. 

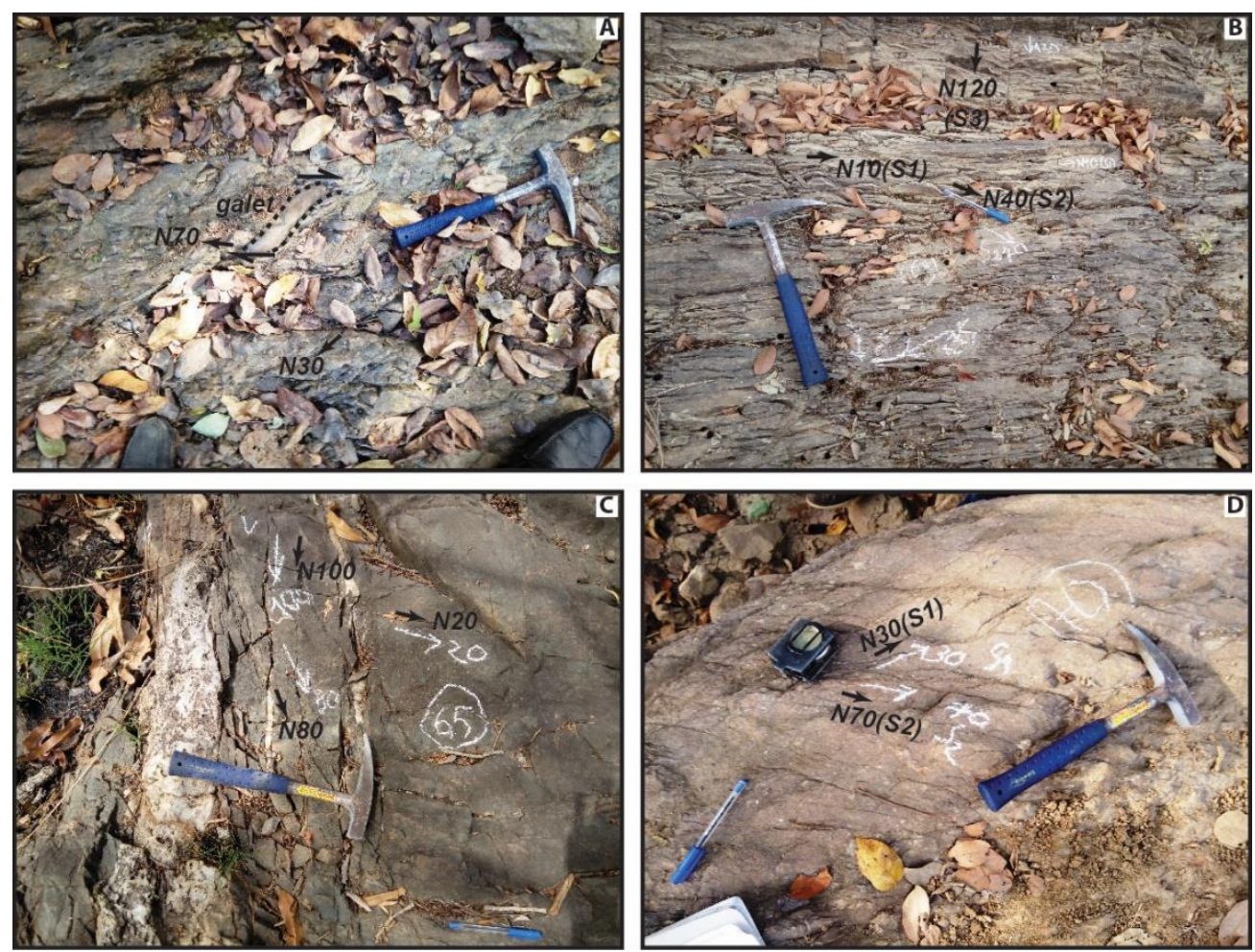

Figure 9. Structures dans la série volcano-sédimentaire. A : Métaconglomérat avec galets de quartz déformés indiquant un cisaillement $\mathrm{N} 70^{\circ}$ dextre ; Micaschiste indiquant les trois schistosités ; C : Roche volcanoclastites avec schistosités de fractures $\mathrm{N} 20^{\circ}$ et $\mathrm{N} 80^{\circ}$, avec des fentes de tension $\mathrm{N} 20^{\circ}$ et $\mathrm{N} 100^{\circ}$; D : Métasédiments avec deux schistosités.

De ce qui précède, au moins trois phases de déformations peuvent être retenues: la déformation D1 crée une foliation dans les métagranites; la déformation D2 qui crée une schistosité de flux et, par la suite, sa crénulation, et un plissement de la foliation; la déformation D3 qui est ductile à cassante.

Une synthèse des données recueillies sur le terrain montre différentes directions des structures (Figures 7 et 10). 

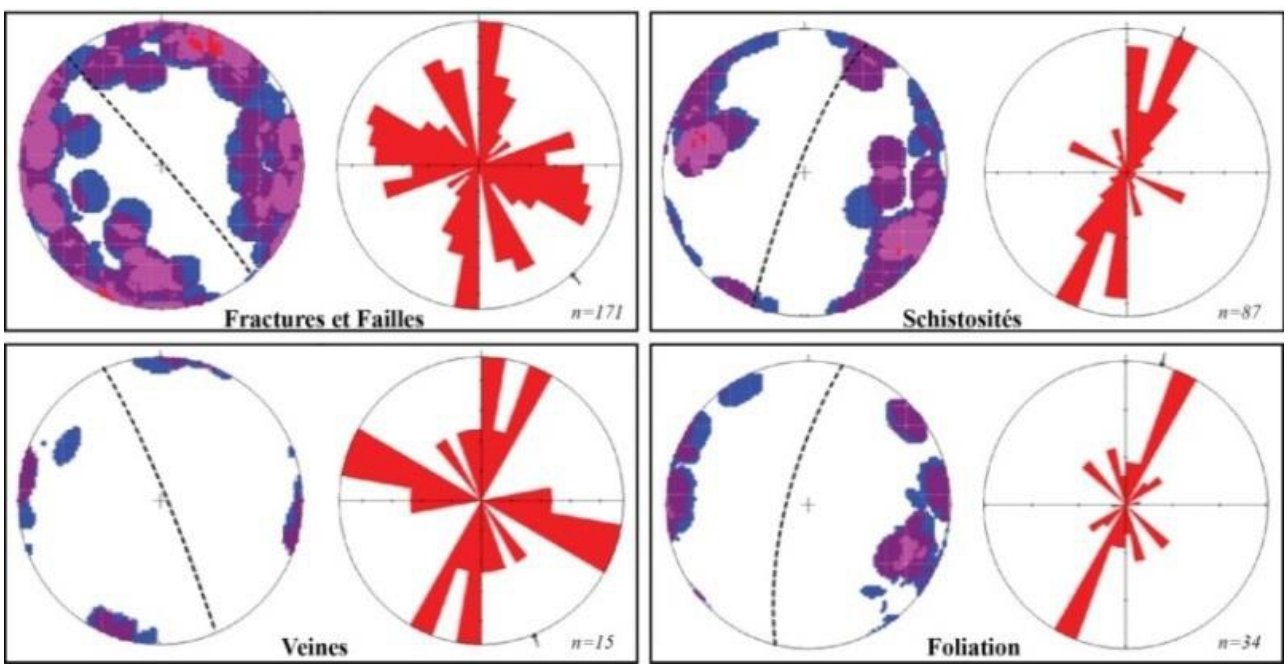

Figure 10. Stéréographes et rosaces directionnelles des structures géologiques mesurées sur le terrain.

\section{Discussion}

Les résultats de nos travaux dans la région de Brobo sont essentiellement structuraux. Les différentes fractures et failles montrent différentes directions. Au niveau de la chronologie, les fractures N-S recoupent les fractures $\mathrm{N} 100^{\circ}$, lesquelles recoupent les fractures $\mathrm{N} 150^{\circ}$. Les fractures $\mathrm{N} 020^{\circ}$ et ${\mathrm{N} 040^{\circ}}^{\circ}$ sont recoupées par celles N-S. Les directions E-O sont les plus récentes.

Sur la base des données recueillies sur le terrain, nous proposons un modèle préliminaire pour expliquer la structuration de la région. (Figure 11):

Les processus géodynamiques se seraient déroulés en trois (3) étapes

Etape 1: Phase d'ouverture de plusieurs bassins. Elle correspond à une distension engendrant une foliation (D1) dans les roches granitiques du socle et s'apparentant à un rifting.

Etape 2 : Phase de fermeture des bassins. Elle est connue en Afrique et se caractérise par une compression NO-SE à NNO-SSE (Milési et al., 1989; Vidal et al., 2009). Cette compression peut avoir des axes globalement E-O à $\mathrm{NE}-\mathrm{SO}$,ce qui fait apparaître des fentes de tension $\mathrm{N} 100^{\circ}$ et $\mathrm{N} 20^{\circ}$. Cette phase est marquée par une déformation des bassins avec formation de plis et de schistosités de flux (déformation D2). Cette phase est suivie, localement, par une schistosité de crénulation dans les métasédiments. Au cours de cette étape, il y a une mise en place de granitoïdes variés, aussi bien dans les ceintures que dans les masses déjà gneissifiées. Des phases de subduction et de collision ne sont pas à exclure. Cette phase de déformation est similaire à la phase Eburnéen II de Grenholm (2014). 
Etape 3 : Phase active des cisaillements ductiles. Au cours de cette étape, plusieurs événements tectoniques se succèdent (D3) : cisaillements ductiles subméridiens issus de la compression NO-SE (failles de N'Zi-Brobo, senestres ou sa réactivation); les failles NE-SO recoupent les premières. Elles sont souvent dextres et responsables de l'amincissement du sillon de Fettêkro, dans la partie sud, ainsi que sa flexion au nord. La structuration se termine par la mise en place d'un certain nombre de structures cassantes à ductiles.

Ce type de modèle de l'évolution tectonique est similaire à ceux décrits en Afrique de l'Ouest. Cependant, le nombre de phases de déformations varie d'un auteur à un autre. Ainsi, Dampare et al. (2009) proposent deux phases de déformations successives au Ghana : une phase de déformation liée aux intrusions granitoïdiques dans les roches birimiennes et au cours de laquelle se forment également des grabens ; ensuite une deuxième phase qui consiste en une déformation progressive liée à une compression régionale NO-SE, engendrant des plis, des chevauchements et des shear zones obliques. Cette compression NO-SE et les différentes phases de déformations qui l'accompagnent est également signalée au Ghana par Feybesse et al. (2006), au Burkina Faso (Tshibubudze et al., 2009 ; Tshibubudze \& Hein, 2010 ; Ouédraogo et al., 2016) et en Côte d'Ivoire (Vidal et al., 1996; Ouattara, 1998 ; Vidal et al., 2009).

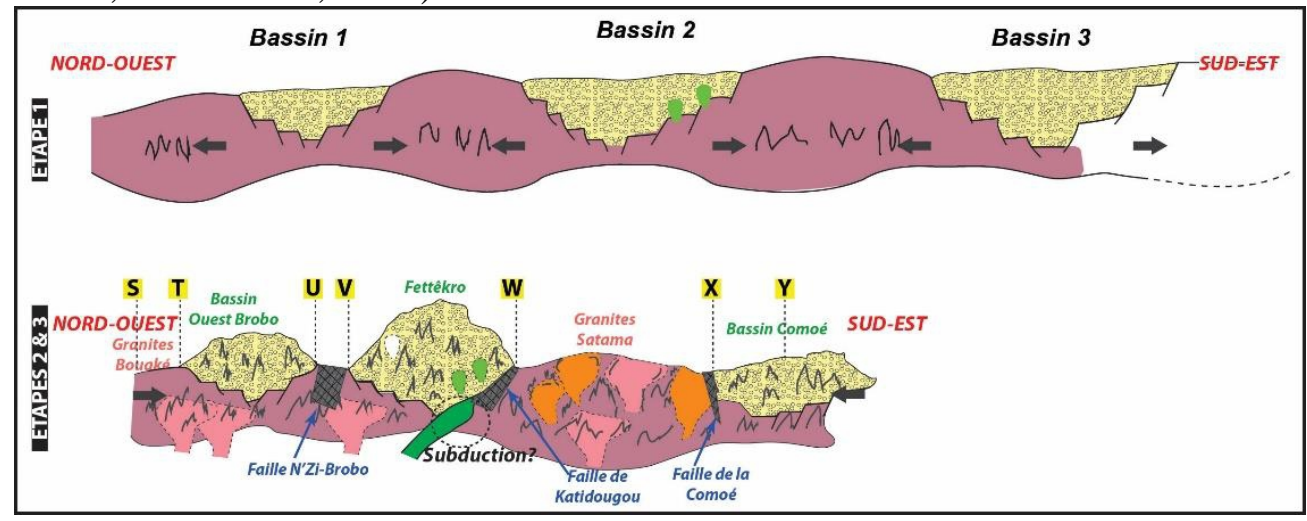

Figure 11. Modèle géodynamique théorique de la structuration de la région de Brobo.

\section{Conclusion}

La région de Brobo est au cœur des terrains paléoprotérozoïques de la Côte d'Ivoire.

Au plan lithologique, plusieurs unités lithologiques ont été distinguées. Ces différentes unités, en fonction de leur lithologie et des structures régionales qui les affectent, correspondent aux métasédiments et roches volcaniques constituant le sillon de Fettêkro et au bassin de la moyenne Comoé, granitoïdes variés.

$\mathrm{Au}$ plan Structural, les principales structures trouvées sont: cisaillements ductiles (N-S senestres, NE-SO dextres, NO-SE dextres ou 
senestres, E-O dextres), schistosités, foliation, plis,... Au moins trois phases de déformations ont affecté les roches (D1, D2 et D3).

Les contextes des minéralisations aurifères décrites dans le sillon d' Oumé (dans la partie Sud) indiquent un contrôle lithologique et structural (Houssou, 2013 ; Ouattara, 2015 ; Houssou et al., 2017). Nombreuses nouvelles zones de failles sont décrites dans la région de Brobo. Celles-ci doivent être considérées par les compagnies minières lorsqu'elles devront faire de l'exploration dans la zone d'étude.

Un modèle géodynamique préliminaire est proposé ? : rifting, puis convergence NO-SE et, enfin, cisaillements ductiles subméridiens. Des travaux complémentaires de géochimie devront permettre de mieux caler les observations de terrain et proposer un modèle final pour cette région.

\section{References:}

1. Adou, M., Gasquet, D. \& Barbey, P. (1990). Crustal growth in the paleoproterozoic from West Africa: the case study of the birimian granitoids from Dabakala area, Ivory Coast. IVth Hulton Symposium, Clermont Ferrand: The origin of granites and related rocks. Doc. BRGM, 290, p. 122.

2. Adou, M. (2000). Cartographie de la feuille Dabakala (Centre -nord de la Côte d'Ivoire) à 1/200 000ème. Nature, âge et origine des granitoïdes. Thèse, INPL, Nancy, $170 \mathrm{p}$.

3. Assié, K.E. (2008). Lode gold mineralization in the Paleoproterozoic (Birimian) volcano-sedimentary sequence of Aféma gold district, southeastern Côte d'Ivoire. Thesis, Faculty of Energy and Economic Sciences Technical, University of Clausthal, Germany, 198 p.

4. Bessoles, B. (1977). Géologie de l'Afrique. Le craton Ouest Africain. Memoires BRGM, Paris, 88, 404 p.

5. Béziat, D., Dubois, M., Debat, P., Nikiéma, S., Salvi, S., \& Tollon, F. (2008). Gold metallogeny in the Birimian craton of Burkina Faso (West Africa). Journal of African Earth Sciences, 50, 215-233.

6. Billa, M. \& Bonnemaison, M. (2009). Geological evolution and metallogeny through the Birimian. Birimian Gold Province 2009 BRGM , Presentation, PDAC, $28 \mathrm{p}$.

7. BRGM (2005). West African GIS Birimian Gold Potential. RC-54093FR-Final report, $44 p$.

8. Dabo, M. (2011). Tectonique et minéralisations aurifères dans les formations birimiennes de Frandi-Boboti, boutonnière de KédougouKéniéba, Sénégal. Thèse de doctorat, Université de Rennes 1, 233 p.

9. Dampare, S., Shibata, T., Asiedu, D., Okano, O., Manu, J., \& Sakyi, P. (2009). $\mathrm{Sr}-\mathrm{Nd}$ isotopic compositions of Paleoproterozoic 
metavolcanic rocks from the southern Ashanti volcanic belt, Ghana. Okayama University, Earth Science Reports, 16, 1; 9-28.

10. Djemai, S., Bendaoud, A., Haddum, H., Ouzegane, K. \& Kienast, J.R. (2009). Apport des images Landsat7 $\mathrm{ETM}^{+}$pour la cartographie géologique des terrains archéens en zone aride : Exemple du terrane de l'In Ouzzal (Hoggar occidentale,Algerie). III'èmes Journées d'Animation Scientifique du réseau de Télédétection de l'AUF JAS'09, Sous le thème: "Imagerie Satellitaire Multisources: Approches Méthodologiques et Applications», Alger, 8-11, novembre 2009.

11. Feybesse, J.-L., Billa, M., Guerrot, C., Duguey, E., \& Bouchot, V. (2006). The paleoproterozoic Ghanaian province: Geodynamic model and ore controls, including regional stress modeling Research. Precambrian Research, 149, 3-4, 149-196.

12. Gnanzou, A., Ouattara, G., Coulibaly, Y., \& Bonin, B. (2015). The Landsat $7 \mathrm{ETM}^{+}$remote sensing imagery for lithological and structural mapping in the central Côte d'Ivoire (West Africa): Case of Dabakala area. European Scientific Journal, 11, 36, 141-163.

13. Gnanzou, A. (2014). Étude des séries volcano-sédimentaires de la région de Dabakala (Nord-Est de la Côte d'Ivoire) : genèse et évolution magmatique: contribution à la connaissance de la minéralisation aurifère de Bobosso dans la série de la Haute-Comoé. Thèse Univ. Paris Sud-Paris XI, 229 p.

14. Grenholm, M. (2014). The Birimian event in the Baoulé Mossi domain (West African Craton) - regional and global context. Dissertations in Geology at Lund University, Master's thesis, no 375 (45 hp/ECTS credits), $116 \mathrm{p}$.

15. Guergour, L. \& Amri, K. (2009). Contribution des images Landsat7 ETM+ à la cartographie géologique et structurale du Bassin de Tin Séririne (Tassilis Oua NAhaggar Hoggar), Algérie. III èmes Journées d'Animation Scientifique du réseau de Télédétection de l'AUF JAS'09, Sous le thème: "Imagerie Satellitaire Multisources: Approches Méthodologiques et Applications», Alger, 8-11 novembre 2009.

16. Himyari, S.M., Hoepffner, C., Benzakour, M., \& Hadani, D.E. (2002). Etude structurale du haut atlas oriental (Maroc) à l'aide de l'analyse linéamentaire des images HRV XS de Spot. Revue Télédétection, 02, 4, 243-253, 2002.

17. Houssou, N.N. (2013). Etude pétrographique, structurale et métallogénique du gisement aurifère d'Agbahou, Divo, Côte d'Ivoire. Doctorat, Univ. Felix HOUPHOUET-BOIGNY, 177 p.

18. Houssou, N.N., Allialy, M.E., Kouadio, F.J.-L.H., \& Gnanzou, A. (2017). Structural Control of Auriferous Mineralization in the 
Birimian: Case of the Agbahou Deposit in the Region of Divo, Côte d'Ivoire. International Journal of Geosciences, 8, 189-204.

19. Jourda, J.P., Djagoua, E.V., Kouamé, K.F., Saley, M.B., Gronayes, C., Achy, J.J., Biémi, J., \& Razack, M. (2006). Identification et cartographie des unités lithologiques et des accidents structuraux majeurs du département de Korhogo (Nord de la Côted'Ivoire): Apport de l'imagerie $\mathrm{ETM}^{+}$de Landsat. Revue Télédétection, 06, 2, 123-142.

20. Koita, M., Jourde, H., Ruelland, D., Koffi, K., Pistre, S., \& Savane, I. (2010). Cartographie des accidents régionaux et identification de leur rôle dans l'hydrodynamique souterraine en zone de socle. Cas de la région de Dimbokro-Bongouanou (Côte d'Ivoire). Hydrol. Sci. J. 55(5), 805-820.

21. Kouamé, K.F., Lasm, T., Saley, M.B., Tonyé, E., Bernier, M., \& Wade, S. (2009). Extraction linéamentaire par morphologie mathématique sur une image RSO de RadarSat-1: application au socle Archéen de la Côte d'Ivoire. III èmes Journées d'Animation Scientifique du réseau de Télédétection de l'AUFJAS'09, Sous le thème: "Imagerie Satellitaire Multisources: Approches Méthodologiques et Applications», Alger, 8-11 novembre 2009.

22. Kusnir (1999). Gold in Mali. Acta Montanistica, 4, 311-318.

23. Leake, M.H. (1992). The petrogenesis and structural evolution of the early Proterozoic Fettekro greenstone belt, Dabakala region, NE Côte d'Ivoire. Unpub. Thesis, Portsmouth, U.K., 315 p.

24. Lemoine, S., Tempier, P., Basso, J.P., Caen-Vachette, M., Vialette Y., Wenmenga, U., \& Touré, S. (1985). The Burkinian, an orogenic cycle, precursor of the Eburnean of West Africa. 13th Colloq. Afr. Geol., St Andrews, Scotland.

25. Lemoine, S. (1988). Evolution géologique de la région de Dabakala (NE de la Côte d'Ivoire) au protérozoïque inférieur. Possibilités d'extension au reste de la Côte d'Ivoire et au Burkina Faso. Thèse ès Sci., Univ. Clermont-Ferrand, 388 p.

26. Milési, J.P., Ledru, P., Feybesse, J.L., Dommanget, A., \& Marcoux, E. (1992). Early Proterozoic ore deposits and tectonics of the Birimian orogenic belt, West Africa. Precambrian Research, 58, 1-4, 305-344.

27. Milési, J.P., Feybesse, J.L., Ledru, P., Dommanget, A., Ouédraogo, M.F., Marcoux, E., Prost, A.E., Vinchon, C., Sylvain, J.P., Johan, V., Tegyey, M., Calvez, J.Y., \& Lagny, Ph. (1989). Les minéralisations aurifères de l'Afrique de l'Ouest. Leur évolution lithostructurale au Protérozoïque inférieur. Chron. Rech. min., Fr., 497 : 3-98.

28. Mortimer, J. (2016). Paleoproterozoic geology of the Toumodi area, Ivory Coast, 1:100,000. Journal of Maps, 12:sup1, 392-400, DOI: 10.1080/17445647.2016.1227732. 
29. Ouattara, G. (1998). Structure du batholite de Ferkéssédougou (secteur de Zuénoula, Côte d'Ivoire). Thèse doctorat, Université d'Orléans, ISTO, Orléans, 291 p.

30. Ouattara, G., Koffi, G.B. \& Yao, K.A. (2012). Contribution des images satellitales Landsat $7 \mathrm{ETM}^{+}$à la cartographie lithostructurale du Centre-Est de la Côte d'Ivoire (Afrique de l'Ouest). International Journal of Innovation and Applied Studies, Vol. 1 No. 1 Nov., 2012, pp. 61-75.

31. Ouattara, A. S., Baka, D., Ouattara, G., \& Nimaga, A. (2016). Mise en évidence de nouvelles cibles de forages à partir de l'analyse de la fracturation du prospect aurifère de Dougbafla-Oumé (Centre-Ouest de la Côte d'Ivoire). European Scientific Journal, 12, 36, 336 - 354.

32. Ouattara, Z. (2015). Caractères lithostratigraphique, structural, géochimique et métallogénique du gisement d'or de Bonikro, sillon birimien de Fettekro, centre-sud de la Côte d'Ivoire. Doctorat, Univ. Felix HOUPHOUET-BOIGNY, 256 p.

33. Ouédraogo, A., Bamba, O., Ouattara, G., Gampine, E., \& Sawadogo, S. (2016). Caractérisations structurales des gîtes aurifères du corridor de Bouboulou-Bouda au Burkina Faso, Afrique de l'Ouest. Afrique SCIENCE 12 (5), 89-104.

34. Ta, M.Y., Lasm, T., Jourda, J.P., Kouamé, K.F., \& Razack, M. (2008). Cartographie des accidents géologiques par imagerie satellitaire Landsat-7 $\mathrm{ETM}^{+}$et analyse des réseaux de fractures du socle précambrien de la région de Bondoukou (Nord-Est de la Côte d'Ivoire). Revue Télédétection, 8, 2, 119-135.

35. Thiéblemont, D., Liégeois, J.P., Fernandez-Alonso, M., Ouabadi, A., Le Gall, B., Maury, R., Jalludin, M., \& Vidal, M. (1987). Les déformations éburnéennes de l'Unité birimienne de la Comoé (Côte d'Ivoire). J. Af. Earth Sci., 6: 141-152.

36. Traoré, D.Y. (2017). Etude métallogénique du district aurifère de Syama (Mali) : analyse comparative de gisements situés sur une même structure lithosphérique éburnéenne. Doctorat, Université Toulouse III - Paul Sabatier. Géologie et métallogénie, 199 p.

37. Tshibubudze, A., Hein, K.A.A., \& Marquis, P. (2009). The Markoye Shear Zone in NE Burkina Faso. Journal of African Earth Sciences 55, 245-256.

38. Tshibubudze, A. \& Hein, K.A.A. (2010). Tectonic evolution of the Oudalan-Gorouol greenstone belt in NE Burkina Faso and Niger, West African craton. Geophysical Research Abstracts, EGU General Assembly 2010, 12, EGU2010-708. 
39. Vidal, M. \& Alric, G. (1987). The Paleoproterozoic (Birimian) of Haute-Comoé, in the West African Craton in Côte d'Ivoire: a transtensional back-arc basin. Precambrian Res., 65 : 207-229.

40. Vidal, M., Delor, C., Pouclet, A., Simeon, Y., \& Alric, G. (1996). Evolution géodynamique de l'Afrique de l'Ouest entre $2.2 \mathrm{Ga}$ et $2 \mathrm{Ga}$ : Le style «Archéen» des ceintures vertes et des ensembles sédimentaires biriniens du nord-est de la Côte-d'Ivoire. Bull. Soc. Géol. France. 167, 307-319.

41. Vidal, M., Gumiaux, C., Cagnard, F., Pouclet, A., Ouattara, G., \& Pichon, M. (2009). Evolution of a Paleoproterozoic "weaktype" orogeny in the Wes $\mathrm{t}$ African Craton (Ivory Coast). Tectonophysics 477, 145-159.

42. Yao, D.B. (1988). Carte géologique de Côte d'Ivoire, feuille de M'Bahiakro à 1/200 000. Ministère des Mines, Direction de la Géologie.

43. Yao, D.B. (1998). Lithostratigraphie et pétrologie des formations birimiennes de Toumodi-Fettekro (Côte-d'Ivoire) : Implication pour l'évolution pour l'évolution crustale du Paléoprotéroïque du craton Ouest-Africain. Thèse doctorat, Université d'Orléans, France, 191 p. 